\title{
Comparative analysis of the main haematological indexes and RNA detection for the diagnosis of SARS-CoV-2 infection
}

Jialin Xiang ${ }^{1+}$, Zuyi Chen ${ }^{1 \dagger}$, Jie Zhou', Di Tian ${ }^{1}$, Xiusheng Ran², Zhimin Zhang ${ }^{3}$, Shi Shi ${ }^{4}$, Daimin Xiao ${ }^{1 *}$ (D) and Yuanzhong Zhou S* $^{*}$

\begin{abstract}
Background: Severe acute respiratory syndrome coronavirus 2 (SARS-CoV-2) infection has become a public health emergency of international concern. SARS-CoV-2 RNA detection is the diagnostic criterion for coronavirus disease 2019 (COVID-19). Nevertheless, RNA detection has many limitations, such as being time-consuming and costprohibitive, and it must be performed in specialized laboratories. Virus antibody detection is a routine method for screening for multiple viruses, but data about SARS-CoV-2 antibody detection are limited.

Method: Throat swabs and blood were collected from 67 suspected SARS-CoV-2 infection patients at the Affiliated Hospital of Zunyi Medical University and Zunyi Fourth People's Hospital isolated observation departments. Throat swab samples were subjected to SARS-CoV-2 RNA detection by real-time PCR. Blood was used subjected to SARSCoV-2 lgG/lgM detection by an enzyme-linked immunosorbent assay (ELISA) and gold immunochromatography assay (GICA). Blood underwent C-reactive protein detection by immunoturbidimetry, and white blood cells, neutrophil percentages and lymphocyte percentages were counted and calculated, respectively. Clinical symptoms, age and lifestyle habits (smoking and drinking) in all patients were recorded. Data were analysed using SPSS version 19. The results were confirmed by $T$ and $x^{2}$ tests; correlations with detection results were analysed by kappa coefficients. Odds ratio (OR) and corrected OR values were analysed by logistic regression. $P<0.05$ was considered statistically significant.

Results: Of the 67 patients included in this study, 26 were SARS-CoV-2 RNA-positive. GICA IgM sensitivity was 50.9\% (13/26), and specificity was 90.2\% (37/41). ELISA IgM sensitivity was 76.9\% (20/26), and specificity was 90.2\% (37/41). ELISA IgG sensitivity was 76.9\% (20/26), and specificity was 95.1\% (39/41). The kappa coefficients between RNA detection and ELISA IgG, ELISA IgM, and GICA IgM results were $0.741(P<0.01), 0.681(P<0.01)$ and $0.430(P<0.01)$, respectively.

(Continued on next page)
\end{abstract}

\footnotetext{
*Correspondence: 372422022@qq.com; 275760363@qq.com

†Jialin Xiang and Zuyi Chen contributed equally to this work.

'Department of Laboratory medicine, Affiliated Hospital of Zunyi Medical University, Zunyi, Guizhou 563000, P.R. China

${ }^{5}$ School of Public Health, Zunyi Medcial University, Zunyi, Guizhou 563000,

P.R. China

Full list of author information is available at the end of the article
}

C C The Author(s). 2020 Open Access This article is licensed under a Creative Commons Attribution 4.0 International License, which permits use, sharing, adaptation, distribution and reproduction in any medium or format, as long as you give appropriate credit to the original author(s) and the source, provide a link to the Creative Commons licence, and indicate if changes were made. The images or other third party material in this article are included in the article's Creative Commons licence, unless indicated otherwise in a credit line to the material. If material is not included in the article's Creative Commons licence and your intended use is not permitted by statutory regulation or exceeds the permitted use, you will need to obtain permission directly from the copyright holder. To view a copy of this licence, visit http://creativecommons.org/licenses/by/4.0/ The Creative Commons Public Domain Dedication waiver (http://creativecommons.org/publicdomain/zero/1.0/) applies to the data made available in this article, unless otherwise stated in a credit line to the data. 
(Continued from previous page)

Conclusion: Among the candidate blood indicators, serum IgG and IgM detected by ELISA had the best consistency and validity when compared with standard RNA detection; these indicators can be used as potential preliminary screening tools to identify those who should undergo nucleic acid detection in laboratories without RNA detection abilities or as a supplement to RNA detection.

Keywords: SARS-CoV-2, Antibody, Real-time PCR, Gold immunochromatography assay, Enzyme-linked immunosorbent assay

The first novel coronavirus pneumonia 2019 case was reported in December 2019 [1, 2], and the pathogen responsible for new novel coronavirus pneumonia 2019 was named novel coronavirus 2019 [3, 4]. Novel coronavirus 2019 was found to be highly homologous with severe acute respiratory syndrome coronavirus (SARS$\mathrm{CoV}$ ) and Middle East respiratory syndrome coronavirus (MERS-CoV) and had strong transmissibility among human hosts. The World Health Organization (WHO) announced that novel coronavirus 2019 infection was a "public health emergency of international concern" on 31 January 2020 [5-7]. On February 11, 2019, the virus responsible for novel coronavirus 2019 was officially named severe acute respiratory syndrome coronavirus 2 (SAR-CoV-2), and new novel coronavirus pneumonia 2019 was officially named coronavirus disease 2019 (COVID-19) by the WHO. As of April 25, 2020, there were 2,856,339 confirmed SAR-CoV-2-positive cases worldwide. SAR-CoV-2 infection outbreaks have become a serious international public health problem.

SARS-CoV-2 RNA detection is the standard method for SARS-CoV-2 infection diagnosis, and real-time PCR is the main method for SARS-CoV-2 RNA detection; however, COVID-19 imaging results and clinical symptoms have been reported to be inconsistent with the RNA detection results [8]. Several reports noted that some SARS-CoV-2-positive patients had to be tested more than once because of inevitable differences in sampling techniques before the cases were confirmed $[9,10]$. SARS-CoV-2 RNA detection is time-consuming and requires specialized working conditions and equipment. Therefore, other faster and easier auxiliary or alternative detection methods are needed for COVID-19 diagnosis. The C-reactive protein (CRP) level, white blood cell count (WBCC), neutrophil percentage (NP) and lymphocyte percentage (LP) are the main auxiliary haematological indexes in COVID-2019 diagnosis [11]. After virus infection, the immune system defends against the virus and produces specific antibodies. Specific IgM antibodies are produced in the early stage after the body is infected; these antibodies can indicate recent infection and disappear rapidly. High-affinity IgG is produced after IgM to elicit long-term immunity. In SARS-infected patient blood, IgM could be detected after 3-6 days and
IgG after 8 days of infection [12,13]. The China National Health and Health Commission issued the Prevention and Control of COVID-19 (Fifth Edition) on February 21, 2020 , suggesting that the presence of SARS-CoV-2 antibodies can assist in diagnosing COVID-19.

Currently, a rapid, haematology-based method for the diagnosis of COVID-19 based on blood samples is being explored in clinical applications. Data about SARS-CoV2 antibody detection specificity and sensitivity are limited, especially data about antibody detection methods, RNA detection methods and main haematological indexes, which are rarely reported. Therefore, in this study, we collected throat swabs and blood samples from suspected COVID-19 patients and tested the for the presence of SARS-CoV-2 RNA and SARS-CoV-2 IgM/ IgG antibodies and analysed the main haematological indexes (CRP, WBCC, NP and LP) to evaluate the utility of different indexes in COVID-19 diagnosis and to provide data to assist in COVID-19 prevention.

\section{Methods \\ Design and cases}

We enrolled a cohort of patients highly suspected of having COVID-2019 from March 1, 2019, to March 31, 2019, at the Affiliated Hospital of Zunyi Medical University. The patient inclusion criteria were as follows: patients who had close contact with a confirmed COVID-2019 patient and fever as well as computed tomography (CT) features according to the guidelines of diagnosis and treatment of COVID-19 [14, 15]. The exclusion criteria were as follows: confirmed negative or positive patients; and patients with no suspected contact history within 2 weeks. Samples were collected approximately 1 week after close contact with a confirmed COVID-2019 patient or clinical symptoms appeared.

To avoid false negative results, throat swab samples for RNA detection were collected at least twice per patient, with a 24-h interval, and a positive result was considered the final RNA test result if a positive result was obtained. Throat swabs were used for SARS-CoV-2 RNA detection, and blood samples were used for SARSCoV-2 IgG/IgM detection and main haematological index (CRP, WBCC, NP and LP) evaluation. Clinical 
symptoms, age and the lifestyle habits of all patients were recorded.

The present study was approved by the Affiliated Hospital of Zunyi Medical University Ethics Committee under approval number KLL-2020-008. Written informed consent was obtained from the patients or their guardians.

\section{SARS-CoV-2 RNA detection}

Throat swabs were collected and transported at $4{ }^{\circ} \mathrm{C}$ in RNA storage solution (Zeesan Biotech, Xiamen, Fujian) for SARS-CoV-2 screening. During sample collection, two swabs were obtained from each patient and placed in a single RNA storage solution container for detection. All samples were detected within $6 \mathrm{~h}$ of sample collection. SARS-CoV-2 RNA was extracted and purified with a "Virus DNA/RNA Extraction Kit (CDC)" (Xi'an Tianlong Science and Technology, Xi'an, China; batch number: 20021410 T014) according to the manufacturer's instructions. SARS-CoV-2 RNA was detected by real-time PCR using the "Novel Coronavirus 2019-nCoV RNA Detection Kit" (Daan Gene, Guangzhou, China; batch number: 2020001; limit of detection: 500 copies $/ \mathrm{mL}$; test results were compared with 3 different concentrations of positive references and 10 negative references) and "Novel Coronavirus 2019-nCoV RNA Detection Kit" (Shanghai GeneoDX Biotech, Shanghai, China; batch number: COV2020008; limit of detection: 500 copies $/ \mathrm{mL}$; test results were compared with 5 different concentrations of positive references and 10 negative references) according to the manufacturers' instructions. Throat swabs were detected by the "Novel coronavirus 2019-nCoV RNA Detection Kit" (Daan Gene, Guangzhou, China). Suspected positive (cycle threshold near the cut-off threshold of the kit) and positive samples were detected by both real-time PCR kits mentioned above. Negative and positive controls were included in every experiment.

Primers and probes for the two SARS-CoV-2 RNA test kits in this study were established by the Chinese Center for Disease Control and Prevention (CDC), and the two kits are listed in the WHO Emergency Use Listing. The human housekeeping, SARS-CoV-2 ORF1ab and $\mathrm{N}$ genes were tested in the same reaction in each sample. If the housekeeping gene was amplified effectively (cut-off value $\leq 32$ ), the sample and reaction were qualified. When the ORF1ab and $\mathrm{N}$ genes in a single sample were amplified effectively (both RNA detection kits used in this study had cut-off values $\leq 40$ ) at the same time, the sample was considered positive. When only the ORF1ab or $\mathrm{N}$ gene in a single sample was amplified effectively, the test was repeated immediately; if the result of the repeated test was consistent with the first test, the sample was considered positive; if not, the sample was considered negative.

\section{SARS-CoV-2 IgG/lgM detection}

Blood serum/plasma samples were used for SARS-CoV2 IgG/IgM detection. Enzyme-linked immunosorbent assay (ELISA) was used for SARS-CoV-2 IgG and IgM detection with a kit (ZHU HAI LIVZON DIAGNOSTICS INC, Guangdong, China; batch number: 2020020308) according to the manufacturer's instructions. A gold immunochromatography assay (GICA) was used for SARS-CoV-2 IgM detection and was performed with a kit (Hotgen, Beijing, China; batch number: 20200204) according to the manufacturer's instructions. Negative and positive controls were included in every experiment.

\section{CRP, WBC, NP and LP detection}

$\mathrm{C}$-reactive protein was detected in blood by immunoturbidimetry (automation equipment: Olympus 5400; Beckman Coulter, California, USA) according to the manufacturer's instructions. White blood cell counts, neutrophil percentages and lymphocyte percentages were counted and calculated, respectively (automation equipment: Sysmex XN 2000; Sysmex, Kobe, Japan) according to the manufacturer's instructions.

Data were analysed using SPSS version 19 (IBM, Armonk, NY, USA). The results were confirmed by $\mathrm{T}$ and $x^{2}$ tests; correlations with the detection results were analysed by Kappa coefficients. Odds ratio (OR) and corrected OR values were analysed by logistic regression. $P<0.05$ was considered statistically significant.

\section{Results}

The mean $(+/-\mathrm{SD})$ age of the patients was $53.82 \pm$ 20.12 years old. Of the 67 patients, $56.7 \%(39 / 67)$ were male, $16.4 \%$ (11/67) smoked, and 14.9\% (10/67) consumed alcoholic beverages. A total of $55.2 \%(37 / 67)$ of the 67 patients had cough, $17.9 \%(12 / 67)$ had muscle pain, and $65.7 \%(44 / 67)$ had dyspnoea. Twenty-six (38.8\%) patients were confirmed to be SARS-CoV-2positive by RNA detection. The demographics of the SARS-CoV-2-positive and SARS-CoV-2-negative patients are shown in Table 1.

Of the 67 patients, 25.4\% (17/67) were GICA IgM positive, 35.8\% (24/67) were ELISA IgM positive, and 32.8\% $(22 / 67)$ were ELISA IgG positive. Compared with the reference ranges of healthy people, $53.7 \%(36 / 67)$ of patients had higher levels of plasma CRP, 53.7\% (36/67) of patients had higher white blood cell counts, $49.3 \%$ (33/67) of patients had higher neutrophil percentages, and 17.9\% (12/ 67) of patients had higher lymphocyte percentages. The GICA IgM, ELISA IgM, ELISA IgG, CRP, WBCC, NP and LP results in SARS-CoV-2-positive as well as SARS-CoV2-negative patients are shown in Table 2. Only the GICA IgM $(P<0.01)$, ELISA IgM $(P<0.01)$, and ELISA IgG $(P<0.01)$ results between SARS-CoV-2 positive and 
Table 1 The demographics and clinical symptoms of the 67 subjects

\begin{tabular}{|c|c|c|c|c|}
\hline Variables & $\begin{array}{l}\text { NAT(-) } \\
(n=41)\end{array}$ & $\begin{array}{l}\text { NAT(+) } \\
(n=26)\end{array}$ & $\begin{array}{l}\text { Total } \\
(n=67)\end{array}$ & $P$ value \\
\hline Age (mean $\pm S D$, years) & $47.15 \pm 18.94$ & $64.35 \pm 17.51$ & $53.82 \pm 20.12$ & 0.000 \\
\hline Sex & n (\%) & n (\%) & n (\%) & \\
\hline Female & $19(46.3)$ & $10(38.5)$ & $29(43.3)$ & 0.526 \\
\hline Male & $22(53.7)$ & $16(61.5)$ & $38(56.7)$ & \\
\hline \multicolumn{5}{|l|}{ Smoking } \\
\hline Never/former smoker & $34(82.9)$ & $22(84.6)$ & $56(83.6)$ & 0.856 \\
\hline Current smoker & $7(17.1)$ & $4(15.4)$ & $11(16.4)$ & \\
\hline \multicolumn{5}{|l|}{ Drinking } \\
\hline Never/former drinker & $37(90.2)$ & $20(76.9)$ & $57(85.1)$ & 0.136 \\
\hline Current drinker & $4(9.8)$ & $6(23.1)$ & $10(14.9)$ & \\
\hline \multicolumn{5}{|l|}{ Cough } \\
\hline No & $25(61.0)$ & $5(19.2)$ & $30(44.8)$ & 0.001 \\
\hline Yes & $16(39.0)$ & $21(80.8)$ & $37(55.2)$ & \\
\hline \multicolumn{5}{|l|}{ Muscle aches } \\
\hline No & $32(78.0)$ & $23(88.5)$ & $55(82.1)$ & 0.279 \\
\hline Yes & $9(22.0)$ & $3(11.5)$ & $12(17.9)$ & \\
\hline \multicolumn{5}{|l|}{ Dyspnoea } \\
\hline No & $16(39.0)$ & $7(26.9)$ & $23(34.3)$ & 0.309 \\
\hline Yes & $25(61.0)$ & $19(73.1)$ & $44(65.7)$ & \\
\hline
\end{tabular}

SD standard deviation, NAT SARS-CoV-2 RNA, NAT(-) SARS-CoV-2 negative, NAT(+) SARS-CoV-2 positive. $\mathrm{n}$ indicates the patient number

negative patients were statistically significant. Details are shown in Table 2.

Based on the RNA detection results, GICA IgM sensitivity was 50.0\% (13/26), and specificity was 90.2\% (37/ 41). ELISA IgM sensitivity was $76.9 \%$ (20/26), specificity was $90.2 \%$ (37/41). ELISA IgG sensitivity was 76.9\% (20/ $26)$, and specificity was $95.1 \%$ (39/41). Details are shown in Table 2.

The GICA positive and negative predictive values were $76.5 \%(13 / 17)$ and $74.0 \%$ (37/50), respectively. The ELISA IgM positive and negative predictive values were $83.3 \%(20 / 24)$ and $86.0 \%$ (37/43), respectively. The ELISA IgG positive and negative predictive values were $90.9 \%(20 / 22)$ and $86.7 \%(39 / 45)$, respectively (calculated from Table 2).

The kappa coefficients between SARS-CoV-2 RNA and GICA IgM, ELISA IgM and ELISA IgG detection were $0.741 \quad(P<0.01), 0.681 \quad(P<0.01)$ and 0.430 $(P<0.01)$, respectively. The kappa coefficients between SARS-CoV-2 RNA detection and CRP $(P>0.05)$, WBCC $(P>0.05)$, NP $(P>0.05)$ and LP $(P>0.05)$ were not consistent. Details are shown in Table 3.

The odds ratio of SARS-CoV-2 RNA positivity in GICA IgM-positive individuals was 9.25 compared with that in GICA IgM-negative individuals (correction value 29.79, $P=0.01$ ). The odds ratio of SARS-CoV-2 RNA positivity in ELISA IgM-positive individuals was 30.83 compared with that in ELISA IgM-negative individuals (correction value 27.09, $P<0.01$ ). The odds ratio of SARS-CoV-2 RNA positivity in ELISA IgG-positive individuals was 65.00 compared with that in ELISA IgGnegative individuals (correction value 84.16, $P<0.01$ ). The odds ratios of SARS-CoV-2 RNA positivity in those with elevated CRP $(P>0.05)$, WBCCs $(P>0.05)$, NPs $(P>0.05)$, LPs $(P>0.05)$ compared with the odds rations in those without elevated values were not consistent. Details are shown in Table 4.

\section{Discussion}

In this study, based on SARS-CoV-2 RNA test results, there were significant differences in the $\operatorname{IgM}$ and $\operatorname{IgG}$ antibody detection results between SARS-CoV-2-positive and SARS-CoV-2-negative patients by ELISA $(P<0.01)$ and GICA $(P<0.01)$ (see Table 2). SARS-CoV-2 IgM and IgG antibody detection methods had high specificity; and ELISA IgG had the highest specificity $(95.1 \%, 39 / 41)$ and the highest sensitivity $(90.2 \%, 37 / 41)$. The positive predictive values $(90.9 \%, 20 / 22)$ and negative predictive values $(86.7 \%, 39 / 45)$ of the ELISA IgM and IgG methods were the highest, while the positive predictive value $(83.3 \%, 20 / 24)$ and negative predictive value $(86.0 \%, 37 / 43)$ of GICA were lower than those obtained by ELISA. Both the ELISA IgG and IgM methods had high sensitivity $(76.9 \%, 20 / 26)$, while the sensitivity of 
Table 2 The haematological test results of the 67 subjects

\begin{tabular}{lcccc}
\hline Variables & NAT(-) & NAT(+) & Total n (\%) & P value \\
\hline Gold immunochromatography assay (IgM) & & \\
Negative & $37(90.2)$ & $13(50.0)$ & $50(74.6)$ & $\mathbf{0 . 0 0 0}$ \\
Positive & $4(9.8)$ & $13(50.0)$ & $17(25.4)$ & \\
Enzyme-linked immunosorbent assay (IgM) & & \\
Negative & $37(90.2)$ & $6(23.1)$ & $43(64.2)$ & $\mathbf{0 . 0 0 0}$ \\
Positive & 4 (9.8) & $20(76.9)$ & $24(35.8)$ & \\
Enzyme-linked immunosorbent assay (IgG) & & \\
Negative & $39(95.1)$ & $6(23.1)$ & $45(67.2)$ & $\mathbf{0 . 0 0 0}$ \\
Positive & $2(4.9)$ & $20(76.9)$ & $22(32.8)$ & \\
C-reactive protein & & & 0.569 \\
Normal & $20(48.8)$ & $11(42.3)$ & $31(46.3)$ & \\
Elevated & $21(51.2)$ & $15(57.7)$ & $36(53.7)$ & \\
White blood cell count & & & 0.335 \\
Normal & $27(65.9)$ & $20(76.9)$ & $47(70.1)$ & \\
Elevated & $14(34.1)$ & $6(23.1)$ & $20(29.9)$ & \\
Neutrophil percentage & & & \\
Normal & $24(58.5)$ & $10(38.5)$ & $34(50.7)$ & \\
Elevated & $17(41.5)$ & $16(61.5)$ & $33(49.3)$ & \\
Lymphocyte percentage & & & \\
Normal & $31(75.6)$ & $24(92.3)$ & $55(82.1)$ & \\
Elevated & $10(24.4)$ & $2(7.7)$ & $12(17.9)$ & \\
\hline
\end{tabular}

NAT SARS-CoV-2 RNA, NAT(-) SARS-CoV-2 negative, NAT(+) SARS-CoV-2 positive. $n$ indicates the patient number. GICA positive and negative predictive values were $13 / 17(76.5 \%)$ and $37 / 50(74.0 \%)$, respectively. ELISA IgM positive and negative predictive values were $20 / 24(83.3 \%)$ and $37 / 43(86.0 \%)$, respectively. ELISA IgG positive and negative predictive values were 20/22 $(90.9 \%)$ and $39 / 45(86.7 \%)$, respectively

GICA was low $(50.9 \%, 13 / 26)$. CRP $(P>0.05)$, WBCC $(P>0.05), \mathrm{NP}(P>0.05)$ and $\operatorname{LP}(P>0.05)$ were not specific.

SARS-CoV-2 is transmitted by respiratory droplets and close contact; it is easily transmitted in all populations [16]. Generally, fever, fatigue and cough are the main manifestations of COVID-19; a small number of patients have symptoms of nasal obstruction, runny nose, and diarrhoea, while some severe patients have obvious dyspnoea [16-18]. In this study, we found that sex, smoking status, and drinking status had no significant association with SARS-CoV-2 infection, but age had a significant association with SARS-CoV-2 infection (shown in Table 1). Although all populations are easily infected by SARS-CoV-2 [16], older people are more likely to be infected. Dyspnoea and muscle pain were not significantly associated with SARS-CoV-2 infection, but cough was significantly associated with SARS-CoV-2 infection (shown in Table 1); cough may be another important early clinical symptom predictive of COVID-19 diagnosis [16]. CRP, the WBCC, the NP and the LP had no significant associations with SARS-CoV-2 infection (all $P>0.05$ ). Fever and CT features were sample screening criteria $[16,18]$, so fever and CT features were not analysed.

Real-time PCR is the most common detection method for SARS-CoV-2 diagnosis [19]. Nevertheless, SARSCoV-2 RNA real-time PCR must be performed in a specific experimental environment (at least a bio-safety level 2 laboratory) that requires specialized experimental equipment and skilled personnel. The SARS-CoV-2 RNA real-time PCR detection method (including RNA extraction) generally requires $3-5 \mathrm{~h}$ [18]. The SARS$\mathrm{CoV}-2$ antibody ELISA generally requires $2-3 \mathrm{~h}$ and does not require a specific experimental environment [17]. The SARS-CoV-2 antibody GICA requires approximately $20 \mathrm{~min}$ and does not require a specific experimental environment [17].

In this study, based on the SARS-CoV-2 RNA test results, the ORs for SARS-CoV-2 RNA positivity in IgM -/IgG-positive and negative patients were calculated. The confirmed OR in the ELISA IgG-positive group compared with that in IgG-negative group was the highest $(\mathrm{OR}=65.00$, corrected $\mathrm{OR}=81.64, P<0.01)$, followed by those in ELISA IgM-positive $(\mathrm{OR}=30.83$, corrected $\mathrm{OR}=27.09, P<0.01)$ and GICA IgM-positive groups $(\mathrm{OR}=9.25$, corrected $\mathrm{OR}=29.79, P<0.01)$. ELISA IgM detection was the most sensitive method in preliminary screening of a high-risk population in this study; CRP $(P>0.05)$, WBCCs $(P>0.05)$, NPs $(P>0.05)$ and LPs $(P>$

Table 3 Kappa coefficients between detection results

\begin{tabular}{|c|c|c|c|c|c|c|c|c|}
\hline & NAT(+) & GICA(+) (IgM) & ELISA(+) (IgM) & ELISA(+) (lgG) & $\mathrm{CRP}(+)$ & WBCC(+) & $\mathrm{NP}(+)$ & $\mathrm{LP}(+)$ \\
\hline$\overline{\mathrm{NAT}(+)}$ & 1.000 & $0.430^{* *}$ & $0.681^{* *}$ & $0.741^{* *}$ & 0.06 & -0.116 & 0.191 & -0.185 \\
\hline $\mathrm{GICA}(+)(\lg M)$ & - & 1.000 & $0.480^{* *}$ & 0.461 & -0.065 & -0.006 & 0.038 & $-0.266^{*}$ \\
\hline ELISA(+) (lgM) & - & - & 1.000 & $0.868^{* *}$ & 0.065 & -0.078 & $0.251^{*}$ & $-0.241^{*}$ \\
\hline ELISA(+) (lgG) & - & - & - & 1.000 & 0.069 & -0.039 & $0.310^{* *}$ & $-0.255^{*}$ \\
\hline $\operatorname{CRP}(+)$ & - & - & - & - & 1.000 & $0.304^{* *}$ & $0.374^{* *}$ & -0.082 \\
\hline WBCC(+) & - & - & - & - & - & 1.000 & $0.309^{* *}$ & -0.034 \\
\hline$N P(+)$ & - & - & - & - & - & - & 1.000 & 0.066 \\
\hline$L P(+)$ & - & - & - & - & - & - & - & 1.000 \\
\hline
\end{tabular}


Table 4 Odds ratios for abnormal laboratory results according to the RNA testing results

\begin{tabular}{|c|c|c|c|c|}
\hline & \multicolumn{2}{|l|}{ Model $1^{a}$} & \multicolumn{2}{|l|}{ Model $2^{b}$} \\
\hline & OR $(95 \% \mathrm{Cl})$ & $p$-value & OR $(95 \% \mathrm{Cl})$ & $p$-value \\
\hline GICA IgM(+) & $9.25(2.55,33.49)$ & 0.001 & $29.79(4.20,211.34)$ & 0.001 \\
\hline ELISA IgM(+) & $30.83(7.78,122.21)$ & 0.000 & $27.09(4.87,150.82)$ & 0.000 \\
\hline ELISA IgG(+) & $65.00(12.01,351.79)$ & 0.000 & $84.16(7.51,943.41)$ & 0.000 \\
\hline $\operatorname{CRP}(+)$ & $1.30(0.48,3.50)$ & 0.605 & $1.08(0.16,7.51)$ & 0.935 \\
\hline WBCC $(+)$ & $0.58(0.19,1.77)$ & 0.337 & $0.54(0.08,3.60)$ & 0.528 \\
\hline $\mathrm{NP}(+)$ & $2.26(0.83,6.17)$ & 0.112 & $5.29(0.77,36.52)$ & 0.091 \\
\hline $\mathrm{LP}(+)$ & $0.26(0.05,1.29)$ & 0.099 & $1.21(0.04,5.25)$ & 0.527 \\
\hline
\end{tabular}

A logistic regression model was used for analysis. Different blood test results were used as dependent variables, and different RNA test results (classified variables) were used as independent variables

ameans not adjusted for covariates

${ }^{b}$ means adjusted for covariates such as age and cough

$\mathrm{Cl}$ confidence interval, + positive

$0.05)$ could not accurately identify high-risk patients. The kappa coefficients between RNA detection and ELISA IgG, ELISA IgM, and GICA IgM detection were 0.741 $(P<0.01), 0.681(P<0.01)$ and $0.430(P<0.01)$, respectively. The consistency between ELISA IgG detection and RNA detection was good, but the consistency between GICA IgM detection and RNA detection was unsatisfactory. The kappa coefficients between RNA detection and the CRP level $(P>0.05)$, WBCC $(P>0.05)$, NP $(P>0.05)$, and LP $(P>0.05)$ were not statistically significant.

Serum samples for antibody detection were easier to collect (lower risk during sample collection) and preserve (the antibody is more stable than RNA) than throat swabs. The ELISA and GICA methods were easier, cheaper and faster than real-time PCR and may be more suitable for primary hospitals. Antibody detection cannot completely replace RNA detection, but it can be auxiliary to RNA detection [19]. ELISA IgG was the best antibody detection method in this study.

There were some limitation in this study. The sample size was small, and it was difficult to ascertain the sample collection time point in early screening. In the future, more clinical manifestations and detection indicators should be compared. Additional studies should be performed to validate the accuracy of SARS-CoV-2 antibody detection.

\section{Conclusions}

Among the candidate blood indicators, the serum levels of IgG and IgM detected by ELISA had the best consistency and validity when compared with standard RNA detection; antibody detection can be used as a potential preliminary screening method to identify those who should undergo nucleic acid detection in laboratories without RNA detection abilities or as a supplement to RNA detection.

\section{Abbreviations}

SARS-CoV-2: Severe acute respiratory syndrome coronavirus 2; SARS: Severe acute respiratory syndrome coronavirus; MERS: Middle East respiratory syndrome coronavirus; COVID-19: Coronavirus disease 2019; ELISA: Enzymelinked immunosorbent assay; GICA: Gold immunochromatography assay; IgM: Immunoglobulin M; IgG: Immunoglobulin G; CRP: C-reactive protein; WBCC: White blood cell count; NP: Neutrophil percentage; LP: Lymphocyte percentage; CT: Computed tomography; CDC: Centers for disease control and prevention; OR: Odds ratio

\section{Acknowledgements}

Thank all the participants in this study.

\section{Authors' contributions}

JX, ZC, DX and YZ conceived and designed the study. JX, ZC, JZ, DT, XR, ZZ, SS, DX and $Y Z$ performed the experiments. DX and $Y Z$ analyzed the data. $Z C$, $D X$ and $Y Z$ wrote the paper. All authors read and approved the final manuscript.

\section{Funding}

Zuyi Chen received funding from the Affiliated Hospital of Zunyi Medical University (201808). Daimin Xiao received funding from the Honghuagang Science and Technology Department (201811).

\section{Availability of data and materials}

The datasets used and/or analysed during the current study are available from the corresponding author on reasonable request.

\section{Ethics approval and consent to participate}

The present study was approved by the Affiliated Hospital of Zunyi Medical University Ethics Committee under approval number was KLL-2020-008. Written informed consent was obtained from all the patients or their legal guardians/representatives.

Consent for publication

Not applicable.

\section{Competing interests}

The authors declare that they have no competing interests.

\section{Author details}

${ }^{1}$ Department of Laboratory medicine, Affiliated Hospital of Zunyi Medical University, Zunyi, Guizhou 563000, P.R. China. ${ }^{2}$ Center for Disease Control and Prevention of Daozhen, Zunyi, Guizhou 563000, P.R. China. ${ }^{3}$ Department of Medical Genetics, Zuyi Medical University, Zunyi, Guizhou 563000, P.R. China. ${ }^{4}$ Department of Laboratory medicine, The Fourth People's Hospital of Zunyi, Zunyi, Guizhou 563000, P.R. China. ${ }^{5}$ School of Public Health, Zunyi Medcial University, Zunyi, Guizhou 563000, P.R. China. 
Received: 29 April 2020 Accepted: 8 October 2020

Published online: 20 October 2020

\section{References}

1. Huang C, Wang Y, Li X, et al. Clinical features of patients infected with 2019 novel coronavirus in Wuhan, China [J]. Lancet. 2020;395(10223):497-506.

2. Wang $C$, Horby P, Hayden FG, et al. A novel coronavirus outbreak of global health concern [J]. Lancet. 2020;395(10223):470-3.

3. Zhu N, Zhang D, Wang W, et al. A novel coronavirus from patients with pneumonia in China, 2019[J]. N Engl J Med. 2020;382(8):727-33.

4. Li Q, Guan X, Wu P, et al. Early transmission dynamics in Wuhan, China, of novel coronavirus-infected pneumonia [J]. N Engl J Med. 2020;382(13): 1199-1207.

5. National Health Committee of the people's Republic of China. WHO: A novel coronavirus infection pneumonia outbreak constitutes an international public health emergency. 2020. http://www.gov.cn/xinwen/2 020-01/30/content_5473224.htm.

6. Hui DS, I Azhar E, Madani T A, et al. The continuing 2019-nCoV epidemic threat of novel coronaviruses to global health - the latest 2019 novel coronavirus outbreak in Wuhan, China [J]. Int J Infect Dis. 2020;91:264-6.

7. Chen H, Guo J, Wang C, et al. Clinical characteristics and intrauterine vertical transmission potential of COVID-19 infection in nine pregnant women: a retrospective review of medical records [J]. Lancet. 2020;395(10226):809-15.

8. Dai W, Zhang H, Yu J, et al. CT imaging and differential diagnosis of COVID19[J]. Can Assoc Radiol J. 2020;71(2):195-200

9. Ai T, Yang Z, Hou H, et al. Correlation of Chest CT and RT-PCR Testing in Coronavirus Disease 2019 (COVID-19) in China: A report of 1014 cases [J]. Radiology. 2020;296(2):E32-E40.

10. Bai $Y, Y a o L$, Wei $T$, et al. Presumed asymptomatic carrier transmission of COVID-19[J]. JAMA. 2020;323(14):1406-7.

11. National Health Committee of the people's Republic of China. Novel coronavirus pneumonia 2019 prevention and control program (Fifth Edition). 2020. http://www.gov.cn/zhengce/zhengceku/2020-02/22/ content_5482010.htm.

12. Lee HK, Lee BH, Seok SH, et al. Production of specific antibodies against SARScoronavirus nucleocapsid protein without cross reactivity with human coronaviruses 229E and OC43. J Vet Sci. 2010:11(2):165-7.

13. Wan ZYZX. Yan XG IFA in testing specific antibody of SARS coronavirus. South China J Prev Med. 2003;29(3):36-7.

14. Jin $\mathrm{YH}$, Cai $\mathrm{L}$, Cheng ZS, et al. A rapid advice guideline for the diagnosis and treatment of 2019 novel coronavirus (2019-nCoV) infected pneumonia (standard version)[J]. Mil Med Res. 2020;7(1):4.

15. WHO. Infection prevention and control during health care when novel coronavirus (nCoV) infection is suspected: interim guidance [S]. Geneva: World Health Organization; 2020.

16. Guan W, Ni Z, Hu Y, et al. Clinical characteristics of coronavirus disease 2019 in China [J]. N Engl J Med. 2020;382(18):1708-20.

17. Li Z, Yi Y, Luo X, et al. Development and clinical application of A rapid lgMIgG combined antibody test for SARS-CoV-2 infection diagnosis [J]. J Med Virol. 2020.

18. Jia $X$, Zhang $P$, Tian $Y$, et al. Clinical significance of IgM and lgG test for diagnosis of highly suspected COVID-19 infection [J]. medRxiv. 2020.

19. Fang $Y$, Zhang $H$, Xie J, et al. Sensitivity of chest CT for COVID-19: comparison to RT-PCR [J]. Radiology. 2020;296(2):E115-17.

\section{Publisher's Note}

Springer Nature remains neutral with regard to jurisdictional claims in published maps and institutional affiliations.

Ready to submit your research? Choose BMC and benefit from:

- fast, convenient online submission

- thorough peer review by experienced researchers in your field

- rapid publication on acceptance

- support for research data, including large and complex data types

- gold Open Access which fosters wider collaboration and increased citations

- maximum visibility for your research: over $100 \mathrm{M}$ website views per year

At $\mathrm{BMC}$, research is always in progress.

Learn more biomedcentral.com/submissions 